treatment costs may appeal to hospital and health authority managers with responsibility for specific budgets, but the findings reported by Dr Muijen et al fail to reflect the true costs associated with the different types of treatment under investigation. Certain items of care are difficult to cost in purely financial terms, such as relatives' time and the capital element of service costs, and these may require special treatment, based on more than one model (McGuire, 1991). There can be no excuse, however, for neglecting relatively straightforward costs which are disproportionately distributed between the experimental and control groups, including the costs incurred by the hospital out-patient service, primarycare services, the criminal-justice system, community agencies and informal carers. In ignoring these, the authors fail to account for costs which are shifted away from the hospital in-patient service but which are borne by other agencies.

Although unacknowledged in the present paper, the cost-benefit analysis promised for future publication is being undertaken in collaboration with the Personal Social Services Research Unit (PSSRU) at the University of Kent, originators of some of the most innovative and compelling work on the economic evaluation of mental-health services (Knapp, 1991). The PSSRU values comprehensiveness above all other virtues in measuring costs (Knapp \& Beecham, 1990), a feature which will certainly be reflected in later papers. It is especially disappointing therefore that the present authors saw fit to draw invalid conclusions based on incomplete cost data at this stage. It is interesting to note that such findings were omitted from a more comprehensive clinical evaluation which appeared simultaneously in another scientific journal.

Beecham, J., Knapp, M. \& Fenyo, A. (1991) Costs, needs and outcomes, Schizophrenia Bulletin, 17, 427-439.

KNAPP, M. (1991) The direct costs of the community care of chronically mentally ill people. In Evaluation of Comprehensive Care of the Mentally III (eds H. Freeman \& J. Henderson), pp. 142-173. London: Gaskell.

- \& Bezснам, J. (1990) Costing mental health services. Psychological Medicine, 20, 893-908.

MCGUIRE, T. G. (1991) Measuring the economic costs of schizophrenia. Schizophrenia Bulletin, 17, 375-388.

PRiSM Team

S. R. WEICH

Institute of Psychiatry

De Crespigny Park

Denmark Hill

London SE5 8AF

Authors' REPLY: The title and text of our paper indicate that the findings are preliminary. Since then, intriguing additional results have emerged from the complete data set. The paper reporting the full clinical results will be ready soon; that on the full cost-benefit analysis will follow later. We are delighted that the definitive full cost-benefit analysis is indeed being done by such experts as Professor Martin Knapp and Jennifer Beecham. Professor Marks recently had the pleasure of publishing a book (Marks \& Scott, 1991) which contained an excellent chapter by Professor Knapp lucidly analysing relevant issues. Dr Weich might wish to add it to the references he cited.

Marks, I. M. \& Scotr, R. P. (1991) Health Care Delivery: Innovations, Impediments, and Implementation. Cambridge: Cambridge University Press.

\author{
Matt MuiJen \\ ISAAC MARKS \\ JOSEPH CONNOLLY
}

Institute of Psychiatry

De Crespigny Park

Denmark Hill

London SE5 8AF

\section{Insight and illusion}

SIR: In our analysis of the Hamilton and Hopkins scales, when comparing imipramine and placebo, we found the Rasch model more appropriate than factor analysis. Dr Evans et al (Journal, August 1992, 161,272 ) fear that our results can induce the illusion of the death of factor analysis.

Hamilton (1977) has made it clear that placebocontrolled trials refer to general dimensions of therapeutic activity. When comparing imipramine with a placebo control in panic disorder we found it meaningful to refer both to a dimension of depression and to a dimension of outcome of general nature in panic disorder. In a cross-national setting we showed that the Rasch model item analysis was superior to factor analysis in evaluating the transferability of these general dimensions (depression and discomfort) across such variables as cultures, age, and sex.

Hamilton (1977) also made it clear that if we wish to know not only whether a drug has any therapeutic effect at all (i.e. better than placebo) but also to find its place within the other established drugs we should include items relevant for describing the clinical profile. In this respect, factor analysis might give an important, multidimensional insight.

The factor-analytic method in our study was similar to that used by Hamilton. This method might be inferior to the method suggested by Dr Evans et al. It would, therefore, be of great importance to 
re-analyse our data in collaboration with Dr Evans and his group (including the alprazolam data) to see whether factor analysis can give us new insight and thereby decrease the fear of illusion. We also hope that the results can be published in this journal.

Hamitton, M. (1977) Standard criteria for clinical assessment in psychopharmacology. In Evaluation of New Drugs in Clinical Psychopharmacology (eds A. Bertelli, G. B. Cassano, P. Castrogiovanni et al), pp. 101-105. Barcelona: J. R. Prous.

Department of Psychiatry

P. BECH

Frederiksborg General Hospital

DK-3400 Hillerod

Denmark

Danish Institute of Educational Research

P. Allerup

Department of Psychiatry

W. MAIER

University of Mainz

Germany

Psychiatric University Clinic

Munich

Germany

Massachusetts General Hospital

Boston

USA

University of Madrid

Spain

M. Albus

P. LAVORI

J. L. Ayuso

\section{The meaning of insight}

SIR: Drs Markova \& Berrios provide an adequate historical account of the search for a definition of insight (Journal, June 1992, 160, 850-860). However, it seems to us that their attempt to be all-inclusive means that their conclusions offer little guidance to the clinical psychiatrist. 'Insight' in clinical psychiatry will not mean the same as in philosophy, and need not mean the same as the psychodynamic use of the term to indicate a deep level of selfknowledge. The authors appear to recognise this, and state that "whether insight itself is adequately defined may not be as important as reliably measuring perhaps only aspects of the concept". Furthermore, they feel that "it would seem appropriate to grade the level of 'insight' ".

In the context of a diagnosed psychiatric disorder, we propose a pragmatic and hierarchical definition of insight whose predictive validity is amenable to testing:
Level 1: the patient is aware of change in perceptual experiences, cognitive processes, emotions, or behaviours

Level 2: the patient has a feeling of disease engendered by these changes

Level 3: the patient gives verbal recognition that the changes causing disease are pathological, i.e. they amount to an illness

Level 4: the patient acts on this in a manner appropriate to his/her intellectual and cultural background by seeking treatment, or complying with treatment, from a psychiatrist.

This schema would seem to us to offer a practical and testable alternative to previous attempts at a clinically useful definition of insight (e.g. McEvoy et al, 1989; David, 1990). It accommodates, for example, the patient with a psychotic disorder who develops a delusional system to account for perceived unpleasant changes, and whose insight is therefore assessed at level 2, and the patient with a neurotic disorder who self-deceives by denying the psychological nature of the illness, and whose insight is therefore assessed at level 3.

By qualifying the definition "in the context of a diagnosis of psychiatric disorder" we exclude the purely neurological conditions described by Drs Markova \& Berrios. It is possible that the study of these conditions will ultimately cast light on the mechanisms of insight, but they have little practical relevance in routine clinical psychiatry.

DAvid, A. S. (1990) Insight and psychosis. British Journal of Psychiatry, 156, 798-808.

McEvoy, J. P., Apperson, L. J., Appelbaum, P. S., et al (1989) Insight in schizophrenia. Its relationship to acute psychopathology. Journal of Nervous and Mental Disease, 177, 42-47.

Department of Psychiatry

Peter RAVEN

RichaRd MULLEN

Institute of Psychiatry

De Crespigny Park

Denmark Hill

London SE5 8AF

\section{Benzodiazepines with ECT}

SIR: In the article by Cohen \& Lawton (Journal, April $1992,160,545-546)$ they mentioned that they gave electroconvulsive therapy (ECT) using an Ectron duopulse constant-current machine at waveform 2 with a stimulus lasting for 1.5 seconds. In the table about stimulus delivered by machines by Russell (1988), a duopulse machine giving waveform 2 for 